\title{
Characterization of cytokinin signaling and homeostasis gene families in two hardwood tree species: Populus trichocarpa and Prunus persica
}

\author{
Juha Immanen ${ }^{1}$, Kaisa Nieminen², Héctor Duchens Silva ${ }^{3}$, Fernanda Rodríguez Rojas ${ }^{4,5}$, Lee A Meisel ${ }^{4,5}$, \\ Herman Silva ${ }^{3}$, Victor A Albert ${ }^{6}$, Torgeir R Hvidsten ${ }^{7,8}$ and Ykä Helariutta ${ }^{{ }^{*}}$
}

\begin{abstract}
Background: Through the diversity of cytokinin regulated processes, this phytohormone has a profound impact on plant growth and development. Cytokinin signaling is involved in the control of apical and lateral meristem activity, branching pattern of the shoot, and leaf senescence. These processes influence several traits, including the stem diameter, shoot architecture, and perennial life cycle, which define the development of woody plants. To facilitate research about the role of cytokinin in regulation of woody plant development, we have identified genes associated with cytokinin signaling and homeostasis pathways from two hardwood tree species.
\end{abstract}

Results: Taking advantage of the sequenced black cottonwood (Populus trichocarpa) and peach (Prunus persica) genomes, we have compiled a comprehensive list of genes involved in these pathways. We identified genes belonging to the six families of cytokinin oxidases (CKXs), isopentenyl transferases (IPTs), LONELY GUY genes (LOGs), two-component receptors, histidine containing phosphotransmitters (HPts), and response regulators (RRs). All together 85 Populus and 45 Prunus genes were identified, and compared to their Arabidopsis orthologs through phylogenetic analyses.

Conclusions: In general, when compared to Arabidopsis, differences in gene family structure were often seen in only one of the two tree species. However, one class of genes associated with cytokinin signal transduction, the CKI1-like family of two-component histidine kinases, was larger in both Populus and Prunus than in Arabidopsis.

Keywords: Cytokinin signaling, Cytokinin homeostasis, Populus trichocarpa, Black cottonwood, Prunus persica, Peach

\section{Background}

Cytokinin signaling contributes to the regulation of multiple fundamental processes active in plant development. These include cell division, meristem maintenance, shoot initiation and growth, vascular patterning, flower and seed development, nutrient uptake, chloroplast differentiation and light perception [1-3]. Additionally, this hormone plays a role in regulating several developmental programs defining the life of perennial woody plants, including the activity of vascular cambium, branching pattern of the shoot, and the onset of leaf senescence. The long life span

\footnotetext{
* Correspondence: yrjo.helariutta@helsinki.fi

'Institute of Biotechnology and Department of Biosciences, University of Helsinki, Fl-00014 Helsinki, Finland

Full list of author information is available at the end of the article
}

and extensive radial growth contribute to the large size and massive amount of wood present in a tree, creating a stark contrast to the much smaller herbaceous annuals. However, only few studies have thus far been published about the role of cytokinin in the regulation of woody plant development. To facilitate this research, we are now presenting a comprehensive description of cytokinin signaling and homeostasis gene families in two hardwood tree species: Populus trichocarpa and Prunus persica. Gene identification in tree genomes was based on homology with Arabidopsis genes, as cytokinin homeostasis and signal transduction pathways have been extensively studied and well-characterized in this species [1-3].

Structurally, cytokinins are adenine derivatives; based on side chain identity they can be classified into four

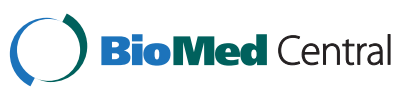


groups representing isopentenyladenine (iP), trans-zeatin $(\mathrm{tZ})$, cis-zeatin, and aromatic cytokinins. iP and $\mathrm{tZ}$ are the bioactive forms of this hormone, to which plants respond through a multistep two-component histidine-aspartate (His-Asp-His-Asp) phosphorelay system [4-6]. The phosphorelay is initiated when a cytokinin ligand binds to a histidine kinase receptor, which triggers autophosphorylation of a His residue. After an intramolecular transfer of the phosphoryl to an Asp residue, it will be transferred to a His in a cytosolic histidine phosphotransfer (HPt) protein. The HPts provide a mobile connection between the cytosol and nucleus; they continuously cycle between these two compartments. In the nucleus, the HPt transfers the phosphoryl onto an Asp in a phospho-accepting response regulator (RRs). RRs can be classified into several different types according to their structure and function. Type-B RRs, which belong to the Myb-transcription factors, activate the transcription of cytokinin primary response genes. Among them are the type-A RRs, which are involved in a negative feedback mechanism that helps to fine-tune the function of cytokinin signaling pathway. Type-A RRs repress activity of type-B RRs [4,7] and are stabilized by HPt mediated phosphorylation (To et al. [8]). Adding further flexibility to the signaling pathway, many of its components are capable of forming both homo- and heterodimers [9-13]. Different combinations of the twocomponent elements presumably add diversity into the process and outcome of the phosphorelay.

Cytokinin signaling represents an ancient hormonal pathway. All of its components are already present in the genome of moss Physcomitrella patens [14,15], indicating that the cytokinin phosphorelay was already functional prior to the development of a well-defined plant vasculature. As compared to the moss, the cytokinin signaling pathway has, however, become more diverse during the evolution of land plants. The number of members in most cytokinin signaling gene families is much higher in the genomes of vascular plants than in Physcomitrella [14,15]. In general, the dynamic nature of plant genomes has influenced the evolution of all gene families in vascular plants. All angiosperm lineages have undergone reoccurring genome duplications, indicating that polyploidization confers a fitness advantage for plant species. Each advent of a whole genome duplication is subsequently followed by a gradual gene loss; this rediploidization ultimately promotes a new duplication, allowing the process to repeat in a cyclical manner [16].

To study the structure of cytokinin signaling and homeostasis genes families in woody plants, we sought to characterize and compare them between two hardwood tree species. For the first species in our phylogenetic study, we chose the most common model tree for molecular biology: Populus trichocarpa, black cottonwood. Populus is a fast growing a dioecious tree, which can reach reproductive maturity in four to six years. Populus trees provide a wood source for the pulp and paper industry and have the potential to be developed into a biofuel feedstock [17]. P. trichocarpa has a relatively small diploid $(2 n=38)$ genome with the haploid size of $485 \mathrm{Mbp}$. The first version of genome assembly was published in 2006 by Tuskan et al. [18]. Due to the challenges of genome assembly in a highly heterozygous tree species, only the current, third genome assembly of $P$. trichocarpa, has been able to resolve a large number of reads that were previously published as unassembled scaffolds. Specific loci identities have only recently been assigned to all predicted genes. Thanks to these improvements, we have now for the first time been able to reliably recover a complete set of cytokinin signaling and homeostasis genes from a tree species. Accordingly, we will discuss how our analysis differs from previously published reports of $P$. trichocarpa cytokinin signaling genes $[14,19,20]$.

The second hardwood tree species used in this study is the economically important fruit tree peach, Prunus persica. In terms of cultivated surface area, P. persica is the third most important temperate fruit crop. Additionally, it is a member of the economically important Rosaceae family, which includes important crops such as peaches, apples, pears, cherries, plums, apricots, strawberries, almonds, and roses. An international effort has led to the genome sequencing and development of Prunus persica as a genomic model for the Rosaceae family [21-23]. This hardwood tree is a self-pollinating diploid $(2 \mathrm{n}=16)$, with a short juvenile period $(2-3$ years $)$ and a genome size of $265 \mathrm{Mbp}$ [22,23].

Currently only a little is known about the role of cytokinin signaling in the regulation of tree or fruit development in Rosaceae. The available data indicates that cytokinins are important for fruit development: high hormone levels have been measured in growing peach fruits [24]. It has also been demonstrated that exogenous application of cytokinin on sweet cherry fruits significantly increases fruit size and weight [25]. Additionally, cytokinin treated fruits showed increased fruit firmness, increased fruit soluble solid concentrations and a delay in exocarp coloration [25]. Similar results have also been seen in apples and pears that have been treated exogenously with cytokinin [26-29]. Taking together, these observations indicate that the cytokinin signaling and homeostasis pathways can provide candidate genes for the breeding of fast growing and high quality Rosaceae fruits.

The third species chosen for our study is the most common herbaceous model plant, Arabidopsis thaliana. Arabidopsis provides an excellent reference genome, as its cytokinin homeostasis and signal transduction pathways have been characterized in detail [1-3]. In addition to the contrast between the woody perennial versus 
herbaceous annual life cycles, the selected three model species differ in their reproductive strategies. Both Prunus and Arabidopsis have hermaphroditic flowers, whereas Populus is a dioecious tree whose genomic sequence was derived from a female plant [18].

All three model species belong to the rosid clade of angiosperm plants. Populus (Malpighiales) and Prunus (Rosales) belong to the eurosids I subclade (Fabidae), whereas Arabidopsis (Brassicales) belongs to the eurosids II (Malvidae) [30]. They display diverse genome duplication histories: since their last common ancestor, Populus lineage has undergone one whole genome duplication, Arabidopis two, and Prunus none [18,31,32]. Based on the genome duplication history and number of synonymous nucleotide substitutions, the molecular-clock rate has been calculated to be faster in Arabidopsis than in Populus [33]. Due to the genome duplication history and gene evolution rate, the Populus genome has on average 1.5 orthologs for each Arabidopsis gene [18], and Prunus 0.85 [34] (http://www.rosaceae.org/projects/peach_genome/v1.0/ homology). The differences in the cytokinin signaling and homeostasis related gene family sizes are consistent with the general genomic trends. We identified a total of 85 genes from the Populus trichocarpa genome and 45 genes from Prunus persica, as compared to the 60 Arabidopsis genes. The gene family structures between the two tree species and Arabidopsis were compared through phylogenetic analyses.

\section{Methods}

\section{Sequence alignments}

Populus and Prunus homologues of Arabidopsis genes were identified by searching the Populus trichocarpa genome database versions 1.1 and 3.0 using various bioinformatic tools and databases available via the Populus genome portal (http://genome.jgi-psf.org/Poptr1_1/Poptr1_ 1.home.html; http://www.phytozome.net/search.php), and the Prunus persica genome version 1 [23], using databases available via The Genome Portal of the Department of Energy Joint Genome Institute [35] (http://genome.jgipsf.org/Poptr1_1/Poptr1_1.home.html), and Phytozome portal [36] (http://www.phytozome.net/search.php?org= Org_Ptrichocarpa_v3.0; http://www.phytozome.net/search. php? method=Org_Ppersica). The bioinformatics tools included BLAST searches, Gene Ontology (GO), Kyoto Encyclopedia of Genes and Genomes (KEGG), EuKaryotic Orthologous Groups Database (KOG) and ortholog finder. Arabidopsis sequences were identified using The Arabidopsis Information Resource (TAIR). Amino acid sequences were aligned using ClustalW followed by manual adjustments when needed [37]. The best bidirectional hit (BBH) method was used as the first approach to determine orthologous pairs of the cytokinin signaling and response genes in Prunus as described by [38]. Protein sequences were aligned using Jalviewand ClustalW2 [39] followed by manual adjustments where needed. The resulting alignment was precisely back-translated to yield a data matrix of the corresponding nucleotide sequences. Gene models, transcript IDs and physical loci of Populus genes used in construction of phylogenetic trees can be found in Additional file 1: Table S1. Gene models and EST support for Prunus genes can be found in Additional file 2: Table S2 (physical loci of the genes are not available in the current 1.0 version of the genome), and TAIR gene numbers of Arabidopsis genes in Additional file 3: Table S3.

\section{Phylogenetic annotation}

We used a maximum likelihood search strategy on amino acid alignments to investigate orthologs and paralogs in the cytokinin signaling and homeostasis gene families. Sequences were aligned using MUSCLE with default settings [40]. A single most optimal tree for each data set was computed using the RaxML BlackBox web server (http:// embnet.vital-it.ch/raxml-bb/) running RaxML version 7.2.8 [41]. Default settings were used with the WAG model of molecular evolution including a gamma parameter. One hundred bootstrap samples were generated to assess support for the inferred relationships. Local bootstrap values (in percentages) are indicated for branches with $\geq 50 \%$ support.

\section{Results and discussion}

To characterize the genetic components of cytokinin signaling and homeostasis pathways from Populus and Prunus, we identified genes belonging to the six families of cytokinin oxidases (CKXs), isopentenyl transferases (IPTs), LONELY GUY genes (LOGs), two-component receptors, histidine containing phosphotransmitters (HPts), and type- $\mathrm{B}$, type- $\mathrm{A}$, and type-C response regulators (RRs). Below we will briefly summarize what is known about each gene family in Arabidopsis, after which we describe them in our two tree species.

\section{Cytokinin oxidases}

Cytokinin oxidase/dehydrogenases (CKXs) are major enzymes responsible for cytokinin catabolism [42,43]. CKX proteins share low sequence homology; the only conserved features are an oxidoreductase FAD-binding domain and a few short consensus motifs [44]. The Populus genome contains eight, Prunus six and Arabidopsis seven CKX genes (Figure 1, Additional file 4: Figure S1). The structure of the gene family is well conserved between all three species (Figure 1).

\section{Isopentenyltransferases (IPTs)}

A major step in cytokinin biosynthesis is catalyzed by ATP/ADP isopentenyltransferases (IPTs), which are 


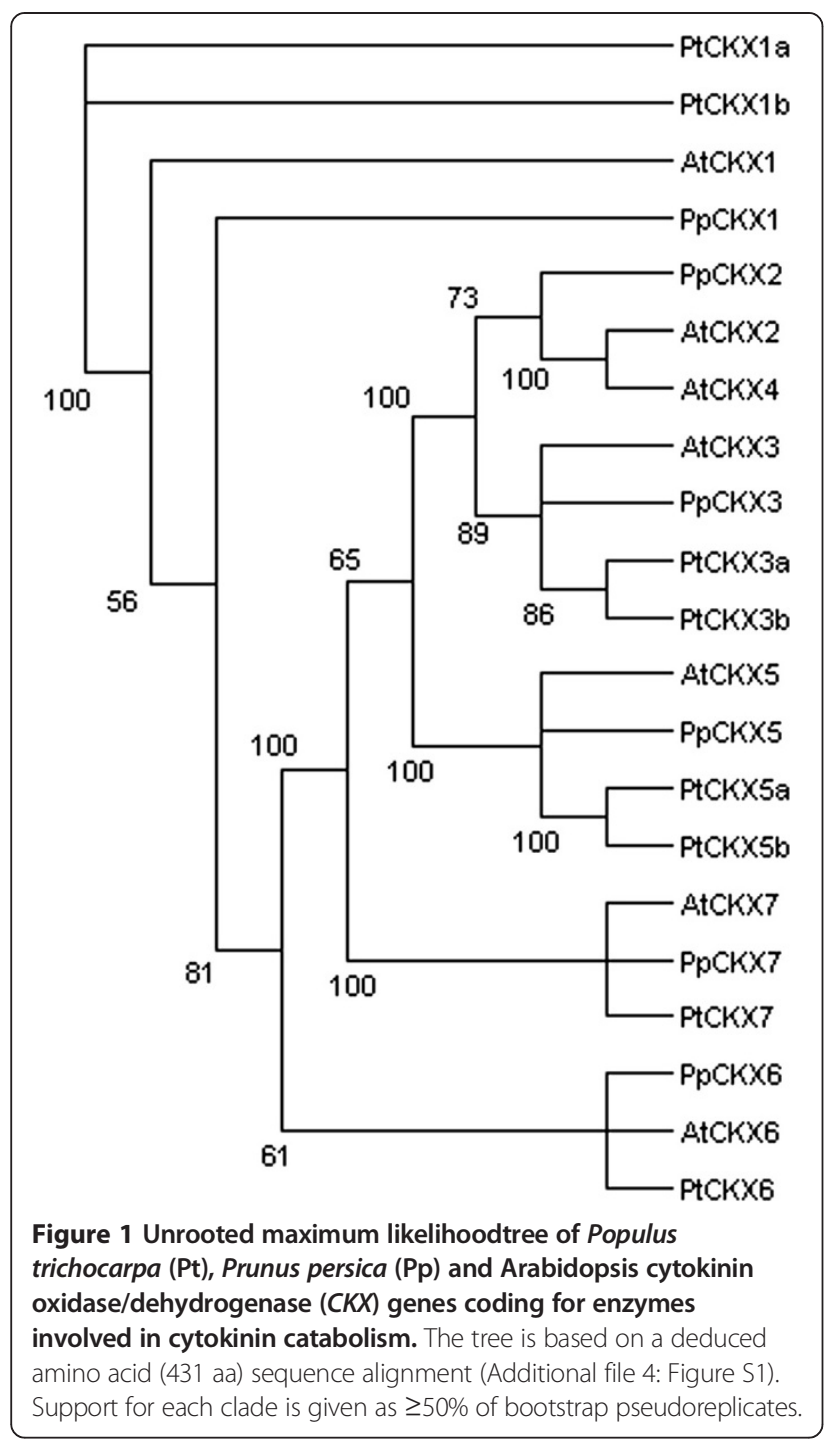

responsible for most of the iP and tZ-type cytokinin biosynthesis [45-49]. They belong to the IPT gene family together with tRNA IPTs, which are responsible for the biosynthesis of cZ-type cytokinins [48]. Both Populus and Arabidopsis genomes contain nine members of the IPT family, whereas Prunus has seven (Figure 2, Additional file 5: Figure S2). Both tree species have one ortholog for each of the two Arabidopsis tRNA IPT genes (IPT2 and IPT9) (Figure 2). The structure of this gene family is otherwise relatively conserved between the three plant species.

\section{LONELY GUY (LOG) genes}

Cytokinin concentration is locally regulated through the activity of LONELY GUY (LOG) enzymes, which convert conjugated cytokinin nucleotides into their bioactive nucleobase forms [50,51]. These enzymes are important regulators of shoot and root apical meristem activity [50-53]. The action of LOGs enables a plant to separate



and define the exact site and time of cytokinin activation, and respectively its perception, apart from that of its biosynthesis. The Populus genome contains 13 genes 
coding for cytokinin activating LOG enzymes, Prunus has seven and Arabidopsis nine (Figure 3, Additional file 6: Figure S3). The number of orthologs appears to have multiplied in one clade in the Populus lineage. This species has four orthologs (PtLOG5a-d) of Arabidopis AtLOG5, whereas Prunus has only one (PpLOG5) (Figure 3).

\section{Two-component receptors}

The initial perception of bioactive cytokinins takes place through CRE1-like two-component histidine kinase receptors, which belong to the superfamily of two-component regulators $[5,54]$. In addition to the cytokinin receptors,

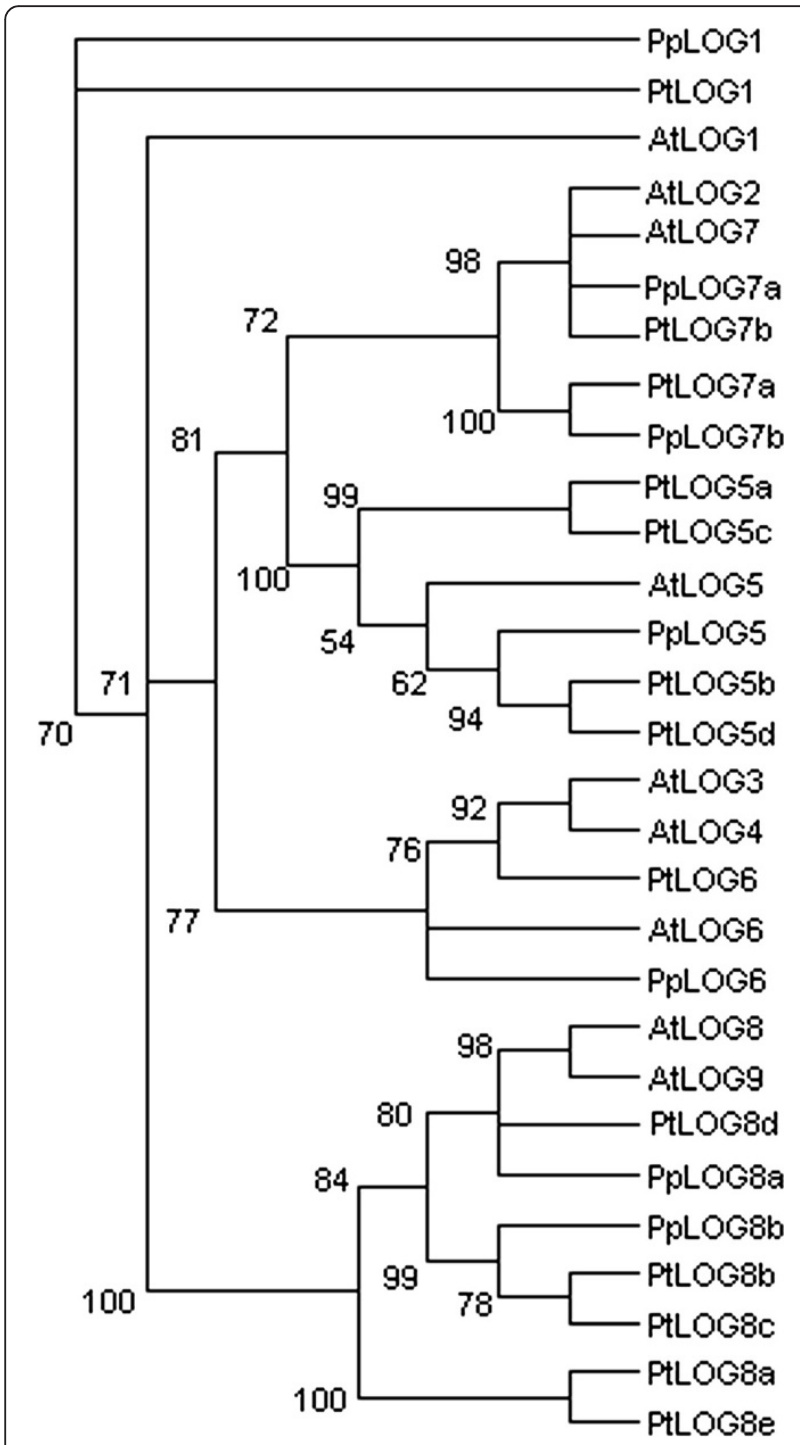

Figure 3 Unrooted maximum likelihood tree of Populus (Pt), Prunus (Pp) and Arabidopsis LONELY GUY (LOG) genes. LOGS convert conjugated cytokinins into their bioactive forms. The tree is based on a deduced amino acid (198 aa) sequence alignment (Additional file 6: Figure S3). Support for each clade is given as $\geq 50 \%$ of bootstrap pseudoreplicates. this family contains a two-component histidine kinase CKI1 that is able to activate the cytokinin phosphorelay [55], five ethylene receptors (ETR1, ETR2, ERS1, ERS2 and EIN4), five phytochromes (PHYA-E), one putative osmosensor (AtHK1), and a histidine kinase (CKI2/AHK5) associated with ethylene and ABA signaling [56-58]. Our focus will be on the CRE1- and CKI1-like subfamilies that are known to participate in cytokinin signaling phosphorelay.

Arabidopsis has three cytokinin receptors: CRE1/WOL/ AHK4, AHK2 and AHK3 [5,54,59]. These receptors have a cytokinin binding CHASE domain, transmembrane domains, a His kinase domain and a receiver domain which contains the phospho-accepting Asp. They share overlapping functions: single null mutants do not have notable phenotypes, whereas the triple mutant is a severely dwarfed and infertile plant [54].

One of the three receptors, CRE1, has both kinase and phosphatase activity: upon binding cytokinin it phosphorylates HPts, whereas in the absence of the hormone it instead dephosphorylates them [6]. Its phosphatase activity helps to quickly inactivate the phosphorelay when the cytokinin levels decrease. In addition to the three canonical receptors, Arabidopsis has a fourth two-component histidine kinase, CKI1, which is capable of inducing cytokinin responses [55]. This kinase can initiate the phosphorelay, but independently of cytokinin [6,60-62]. As it is missing the cytokinin binding CHASE domain, it does not represent a true cytokinin receptor. Further in contrast to the CRE1-like receptors, which are mainly located at the endoplasmic reticulum [11,63], CKI1 appears to be present at the plasma membrane [4,64].

The Populus genome contains five cytokinin receptor genes (PtCRE1a, PtCRE1b, PtHK2, PtHK3a and PtHK3b) [19], and Prunus three (PpCRE1,PpHK2, PpHK3), all orthologous to the three Arabidopsis CRE1-like receptors (Figure 4, Additional file 7: Figure S4). In contrast, both tree species have three orthologs of CKI1 (PtCKI1a-c; $P p C K I 1 a-c$ ), a single copy gene in Arabidopsis (Figure 4, Additional file 7: Figure S4). The significant (3-fold) expansion of the CKI1 gene family appears to be specific for the Populus and Prunus lineages, as both soybean [65] and rice [66] are similar to Arabidiopsis, having only one ortholog of this gene. CKI1 is known to be essential for female gametophyte development [67], and interestingly, has also been reported to regulate vascular development in Arabidopsis inflorescence stem. In the study by Hejátko et al. [64], CKI1 expression was detected in vascular tissues, and its over-expression increased the number of vascular cambial cells in vascular bundles. Accordingly, the number of cambial cells was reduced in RNAi lines where the CKI1 expression level was down-regulated [64]. Therefore, CKI1 appears to have a stimulatory role in regulation of vascular cell proliferation in Arabidopsis. 


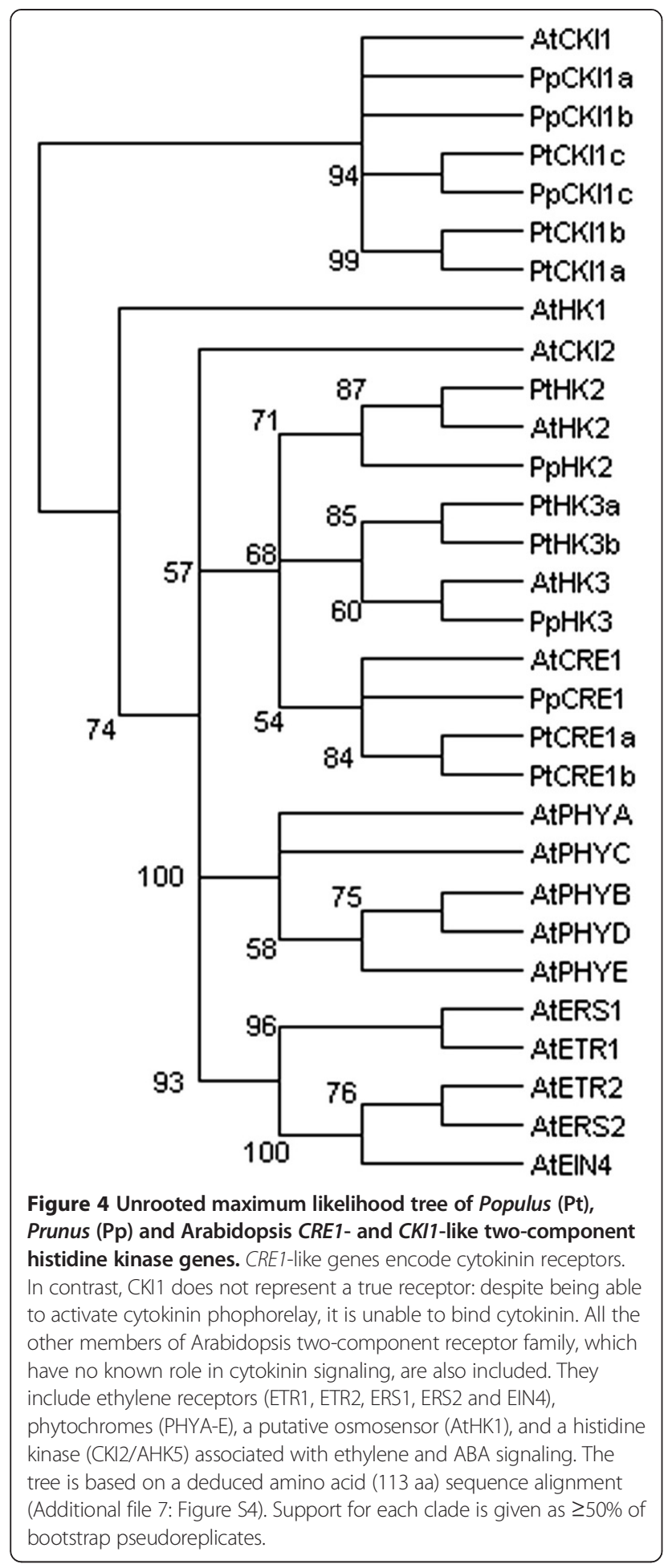

Histidine containing phosphotransmitters

Upon binding cytokinin, the CRE1-like receptors initiate phosphorylation of histidine containing phosphotransmitters (HPts), which are continuously cycling between cytosol and nucleus [12]. This movement enables the transfer of phosphoryl groups from the membrane-localized receptors to the nuclear-localized response regulators.

The HPts are characterized by a short motif, HQxKGSSxS, which contains a conserved phospho-accepting His residue (Additional file 8: Figure S5) [68]. In Arabidopsis, five members of the gene family (AHP1-5) contain this canonical consensus motif [69-72]. They share partially redundant functions since higher-order null mutants, that are lacking multiple genes from the same gene family, display a progressively reduced sensitivity to cytokinin [73].

In contrast to the five canonical members, two Arabidopsis HPt genes, AHP6 and AHP-like (At4g04402), contain an atypical motif lacking the conserved His residue [71]. AHP6 has an inhibitory role on the cytokinin phosphorelay, and has been classified as a pseudo HPt [74]. AHP6 negatively interferes with the phosphorelay, potentially by competing with the other AHPs for interaction with phoshorylated receptors. In Arabidopsis roots, the expression of AHP6 promotes differentiation of protoxylem, the first xylem cell type that forms in a developing vasculature [74]. The negative function of AHP6 contributes to the generation of distinct and well-defined domains of low cytokinin signaling. The function and expression pattern of the AHP-like gene is not known.

All together 14 HPt-encoding genes were identified in the new Populus genome assembly (Figure 5, Additional file 8: Figure S5); four more than were reported by Pils and Heyl [14]. Nine HPts were identified in Prunus, as compared to the seven in Arabidopsis (Figure 5, Additional file 8: Figure S5). The gene family structure is relatively different between the tree species and Arabidopsis (Figure 5). The trees have one clade (PtHP8a, PtHP8b and PpHP8) with no evident Arabidopsis orthologs, and Populus has one more (PtHP1a and PtHP1b) without either an Arabidopsis or Prunus ortholog. Two Populus (PtHP6a, PtHP6b) and one Prunus HPt (PpHP6) are orthologous to the Arabidopsis pseudo HPt AHP6, and accordingly lack the phospho-accepting His residue (Additional file 8: Figure S5). One Populus (PtHP-like) and one Prunus gene (PtHP-like) contain non-canonical consensus motifs lacking the conserved histidines (Additional file 8: Figure S5); it is not known if these proteins participate in the phosphorelay.

Interestingly, both Populus and Prunus have four orthologs of a single Arabidopsis gene, AHP4. However, this is the case also in two monocot species; rice and maize, which both have three orthologs of this gene [73,75,76]. Compared to other species, it appears that Arabidopsis has lost its AHP4 homologs during evolution. Somewhat surprisingly, despite the loss of these potentially redundant genes, the phenotype of an Arabidopsis AHP4 null mutant is not very striking. In Arabidopsis, $A H P 4$ is primarily expressed in young flowers, and the null mutant shows slightly more secondary cell wall thickening at some 


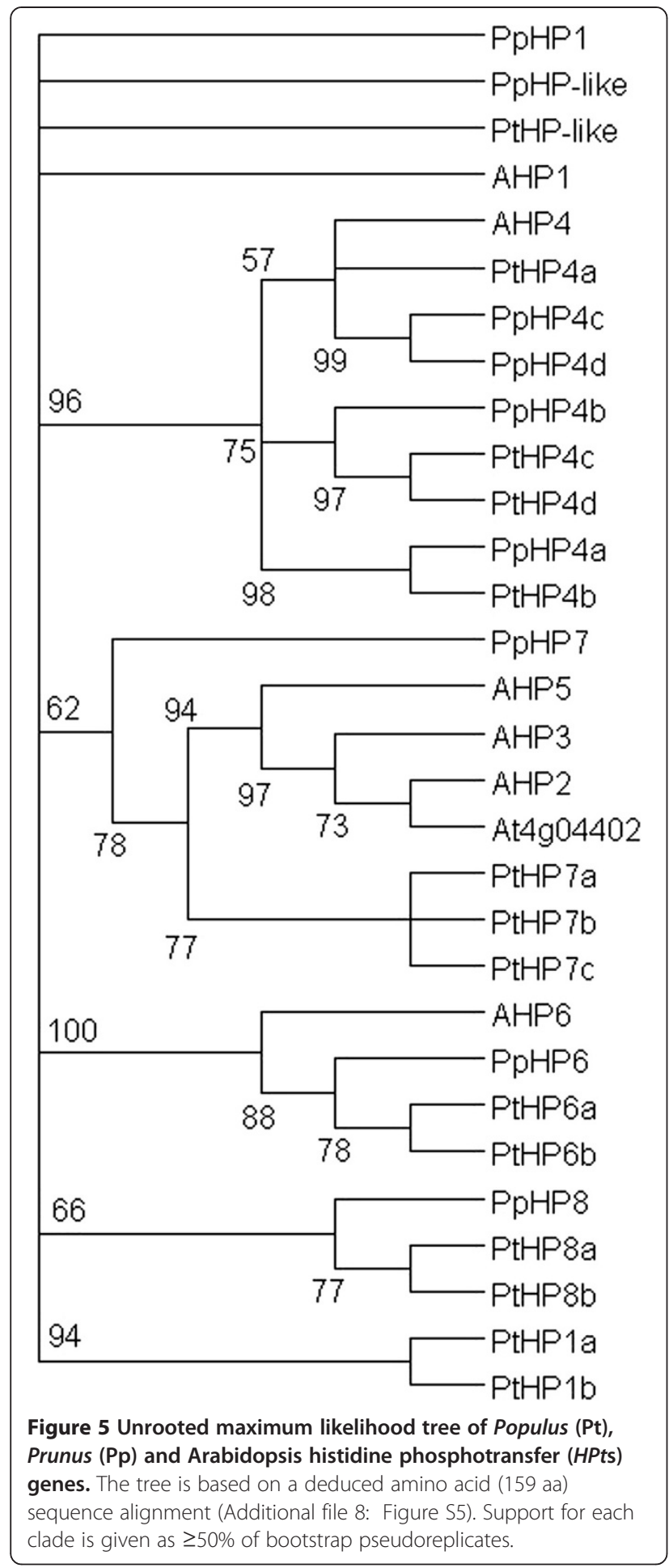

anther tissues; yet its fertility is not impaired [77]. Presumably the AHP4 function is still redundant with the other Arabidopsis HPt proteins. One Populus AHP4 ortholog (PtHP4b) is missing the conserved phospho-accepting His residue. This reflects the situation in monocots, where all three rice AHP4 orthologs [73], and two from the three maize orthologs, also lack the conserved histidine [76]. These genes appear to have evolved into pseudo-response regulators with a potentially inhibitory role on the cytokinin signaling phosphorelay.

\section{Response regulators}

Response regulators (RRs) represent the final components of the cytokinin signaling phosphorelay. The common feature of all RRs is a receiver domain, which contains the phospho-accepting Asp residue as part of the core sequence DD-D-K (Asp Asp-Asp-Lys) [56,57]. The RRs can be classified into four subfamilies: A-type RRs with the receiver domain; B-type RRs with the receiver domain fused to a DNA-binding (GARP) sequence; C-type RRs, which despite an atypical amino acid sequence of their receiver domain, still contain the phospho-accepting Asp residue; and pseudo RRs lacking the conserved Asp in their receiver domain. Members of the type-A, -B and -C RR subfamilies participate in the cytokinin signaling phoshorelay $[56,57,78]$. The pseudo RRs are, instead of cytokinin signaling, known to function in the regulation of light responses, including circadian rhythms [79-81]. We will not discuss them in this article.

\section{Type-B RRs}

Type-B RRs are DNA-binding transcriptional regulators that positively mediate cytokinin responses [4,82-84]. They activate transcription of cytokinin primary response genes; among them the type-A RRs. The expression of type-B RRs themselves is not induced through cytokinin signaling; their activity is regulated through phosphorylation of a conserved Asp residue in the receiver domain. At least one Arabidopsis B-type RR, ARR2, is rapidly degraded upon its cytokinin induced phophorylation [85]. This mechanism presumably provides proteolysis mediated feedback regulation for its activity. In Arabidopsis, the type-B RRs share partially redundant functions; higher order null mutants show a progressively decreased sensitivity to cytokinin $[84,86,87]$.

There are six type-B RR genes in Prunus genome, whereas Populus has thirteen (PtRR13-25) and Arabidopsis have both twelve [88] (Figure 6, Additional file 9: Figure S6). Two of the Arabidopsis (ARR18 and ARR23) genes however code for a truncated form of the receiver domain, thus their functionality as RRs is questionable. The structure of Populus RR family has previously been reported by Ramírez-Carvajal [20] and Pils and Heyl [14]. In these two reports, altogether 13 type-B Populus RRs (PtRR12-23) were identified, from which PtRR12 is missing from the current assembly, whereas PtRR24 represents a newly identified gene. 
Figure 6 Unrooted maximum likelihood tree of Populus (Pt), Prunus (Pp) and Arabidopsis response regulators (RRs).

Members of type-A, $-B$ and $-C$ RRs are involved in cytokinin signaling. For Arabidopsis, also the pseudo response regulator genes with no known role in cytokinin signaling are included. The tree is based on a deduced amino acid (226 aa) sequence alignment for receiver (all RRs) and DNA-binding (GARP) (B-type RRs) domains (Additional file 9: Figure S6). Support for each clade is given as $\geq 50 \%$ of bootstrap pseudoreplicates.

\section{Type-A RRs}

The promoters of type-A RR genes contain a high number of B-type RR binding sites [82,83,89,90]. Accordingly, phosphorylated type-B RRs activate the expression of type-A RR genes, which function as negative feedback regulators of cytokinin signaling [8,91-93]. Type-A RRs may repress the type-B RR activity either by competing with them for phosphotransfer from upstream HPts or by forming inactive heterodimers with them [94]. Similar to the B-type, in general, individual A-type RRs are also redundant in Arabidopsis; sensitivity to cytokinin increases progressively in higher order mutants $[86,87,91])$. Nevertheless, there are also phenotypic differences between Arabidopsis mutants lacking multiple type-A RRs in different combinations. At least two RRs often share highly redundant functions, but these functions differ somewhat from those shared between the other gene pairs [8,91-93]. It seems likely that some functional specificity has evolved between the different RR genes and contributes to their retention in plant genomes.

The Populus genome contains eleven types-A RRs (PtRR1-11), whereas Prunus has only four, compared to the ten genes present in the Arabidopsis (Figure 6, Additional file 9: Figure S6). Both Ramírez-Carvajal et al. [20] and Pils and Heyl [14] identified these same type-A Populus RRs (Figure 6). It appears that Prunus has lost members of this subfamily during its evolution. However, based on the relatively high redundancy between Arabidopsis RRs, this gene family appears to be well buffered against loss of individual genes.

\section{Type-C RRs}

Type-C, or extra, RRs represent a response regulator subfamily characterized by an atypical receiver domain amino acid sequence [80]. Arabidopsis has two of them, ARR22 and ARR24. They display very restricted expression patterns: ARR22 is expressed exclusively in developing seeds [95], and ARR24 only in developing and mature pollen grains [96]. ARR22 can interact and dephosphorylate HPt proteins in vitro; it thus appears to represent a negative regulator of the cytokinin signaling phosphorelay $[78,95]$. In contrast to the type-A RRs, the expression of type-C RRs is not cytokinin inducible $[78,96]$. Ectopic over-expression of ARR22 gives rise to a distinct phenotype; the plants are dwarf and sterile [78]. 
Yet surprisingly, neither a vegetative nor a reproductive phenotype was detected in either single or double null mutants of these two genes [95,96]. The function of type-C RRs remains elusive.

Compared to the two Arabidopsis genes, Populus has eight type-C RRs (PtARR26-33), whereas interestingly, none was found in the Prunus genome (Figure 6, Additional file 9: Figure S6). Pils and Heyl [14] identified 10 unnamed typeC Populus RRs, four of which have been removed from the new assembly; whereas PtRR28 and PtRR33 represent newly identified genes on our list. The expansion of typeC subfamily appears to be specific for the evolution of Populus lineage, as we know that several other species have less of them: rice has only two, and both maize and soybean have three [65]. All eight Populus type-C RRs appear to share a common ancestor with the two Arabidopsis extra RRs. Two of them, PtRR27 and PtRR29, have an atypical conserved motif (HD-D-K and DD-E-K, respectively), and may represent pseudo response regulators.

\section{Conclusions}

We report here the first comprehensive description of cytokinin signaling and homeostasis gene families in two hardwood tree species; Populus trichocarpa and Prunus persica. Genomes of both species contain the same cytokinin signal transduction components as Arabidopsis, reflecting the ancient origin of this hormone signaling system. In general, the identified gene families were larger in Populus and smaller in Prunus when compared to Arabidopsis.

In contrast to the consensus ratios, some cytokinin signaling and homeostasis gene families have distinctively expanded in one or two of the tree species as compared to Arabidopsis. One of the expanded clades is the CKI1-like subfamily of two-component histidine kinases. This family has three members in both Populus and Prunus, as compared to a single gene in Arabidopsis. This shared expansion indicates that the gene number has probably been multiplied in a common ancestor of the two tree species. Interestingly, in Arabidopsis CKI1 has been shown to participate in the regulation of both reproductive and secondary vascular development. Future research will show if the extra CKI1 orthologs have any role in the control of cambial activity and wood production in tree species.

Another difference is seen in the HPt gene family, where both tree species have four homologs of the single Arabidopsis AHP4 gene. Yet, in this case, several other species also have multiple AHP4 homologs present in their genomes. Some of these homologs appear to represent pseudo HPts, which potentially act to inhibit the cytokinin phosphorelay. It seems that that there has been no tree lineage specific expansion, but that Arabidopsis has instead lost all but one of its AHP4 homologs.
In contrast to the changes shared by both tree species, some gene expansions appear to have taken place only in the Populus lineage. One clade of the LOG gene family, the Populus orthologs of Arabidopsis AtLOG5, has expanded four-fold as compared to either of the two other species. Another gene subfamily, the C-type RRs, has multiplied four-fold in the Populus lineage as compared to Arabidopsis, but has instead disappeared from Prunus. Possibly other RRs have replaced function of this RR class in Prunus. As the function of C-type RRs has remained elusive in Arabidopsis, Populus could potentially turn out to be a better model for studying their activity.

We hope that the identification of cytokinin signaling and homeostasis pathway from two hardwood tree species may serve as a reference upon which functional analyses can be developed to determine the role that cytokinin plays in vegetative and reproductive tree development. Additionally, these genes may serve as potential candidate genes for marker-assisted breeding towards increased wood and fruit production.

\section{Additional files}

\begin{abstract}
Additional file 1: Table S1. Genetic loci and gene models (transcript IDs) of Populus trichocarpa cytokinin signaling and homeostasis genes based on the genome release version 3.0 (http://www.phytozome.net/ search.php?org=Org_Ptrichocarpa_v3.0). To enable comparisons with previously published Populus gene reports, we have additionally included the respective loci and gene models as they were given in the assembly version 1.1.
\end{abstract}

Additional file 2: Table S2. Gene models and EST support for the Prunus persica cytokinin signaling and homeostasis genes. The gene models are given as in the genome release version 1 (http://www. phytozome.net/search.php?method=Org_Ppersica).

Additional file 3: Table S3. List of Arabidopsis genes used in the construction of the phylogenetic trees.

Additional file 4: Figure S1. Alignment of Populus trichocarpa (Pt), Prunus persica (Pp) and Arabidopsis cytokinin oxidase/dehydrogenases (CKXs)

Additional file 5: Figure S2. Alignment of Populus (Pt), Prunus (Pp) and Arabidopsis isopentenyl transferases (IPTs).

Additional file 6: Figure S3. Alignment of Populus (Pt), Prunus (Pp) and Arabidopsis LONELY GUY (LOG) proteins).

Additional file 7: Figure S4. Alignment of Populus (Pt), Prunus (Pp) and Arabidopsis CRE1- and CKI1-like two-component histidine kinase, together with Arabidopsis ethylene receptors (ETR1, ETR2, ERS1, ERS2 and EIN4), phytochromes (PHYA-E), a putative osmosensor (AtHK1), and the histidine kinase CKI2/AHK5.

Additional file 8: Figure S5. Alignment of Populus (Pt), Prunus (Pp) and Arabidopsis histidine phosphotransfer proteins (HPts). The consensus HQXKGSSXS motif, containing the phospho-accepting histidine residue $(\mathrm{H})$, is marked above the alignment. Altogether four Populus (PtHPt6a, PtHP6b, PHP4b, and PtHP-like), and two Prunus (PpHP6 and PpHP-like) HPts lack the conserved histidine residue.

Additional file 9: Figure S6. Alignment of Populus (Pt), Prunus (Pp) and Arabidopsis response regulators (RRs).

\section{Competing interests}

The authors declare that they have no competing interests. 


\section{Authors' contributions}

$\mathrm{JI}$ and $\mathrm{KN}$ participated in the design of the study, in the gene identification from Populus and Prunus genomes and in the sequence alignment. HDS and FRR participated in the gene identification from Prunus genome and in the sequence alignment. VAA performed the phylogenetic analyses. TRH participated in the design of the study. LAM, HS and YH conceived of the study, and participated in its design and coordination. All authors read and approved the final manuscript.

\section{Acknowledgements}

This work was funded by a collaborative initiative between the Finnish and Chilean Governments (SusEn by Academy of Finland and CONICYT CCF-01). $L M$ received additional funding from CONICYT, FONDECYT/Regular No1121021.

\section{Author details \\ ${ }^{1}$ Institute of Biotechnology and Department of Biosciences, University of Helsinki, FI-00014 Helsinki, Finland. ${ }^{2}$ Finnish Forest Research Institute, Vantaa Research Unit, Jokiniemenkuja 1, Fl-01301 Vantaa, Finland. ${ }^{3}$ Departamento de Producción Agrícola, Laboratorio de Genómica Funcional \& Bioinformática, Universidad de Chile, Facultad de Ciencias Agronómicas, Av. Santa Rosa 11315, 8820808 La Pintana, Santiago, Chile. ${ }^{4}$ Universidad Andres Bello, Centro de Biotecnología Vegetal, Facultad de Ciencias Biológicas, República 217, 837-0146 Santiago, Chile. ${ }^{5}$ Universidad de Chile, Instituto de Nutrición y Tecnología de los Alimentos (INTA), El Líbano 5524, 7830490 Macul, Santiago, Chile. ${ }^{6}$ Department of Biological Sciences, University at Buffalo, Buffalo, NY 14260, USA. ${ }^{7}$ Umeå Plant Science Centre, Department of Plant Physiology, Umeå University, 90187 Umeå, Sweden. ${ }^{8}$ Department of Chemistry, Biotechnology and Food Science, Norwegian University of Life Sciences, 1432 Ås, Norway.}

Received: 24 July 2013 Accepted: 27 November 2013 Published: 16 December 2013

\section{References}

1. Sakakibara H: Cytokinins: activity, biosynthesis, and translocation. Annu Rev Plant Biol 2006, 57:431-449.

2. Durbak $A$, Yao H, McSteen P: Hormone signaling in plant development. Curr Opin Plant Biol 2012, 15:92-96

3. Hwang I, Sheen J, Müller B: Cytokinin signaling networks. Annu Rev Plant Biol 2012, 63:353-380.

4. Hwang I, Sheen J: Two-component circuitry in Arabidopsis cytokinin signal transduction. Nature 2001, 413:383-389.

5. Inoue T, Higuchi M, Hashimoto $Y$, Seki M, Kobayashi M, Kato T, Tabata S, Shinozaki K, Kakimoto T: Identification of CRE1 as a cytokinin receptor from Arabidopsis. Nature 2001, 409:1060-1063.

6. Mähönen AP, Higuchi M, Törmäkangas K, Miyawaki K, Pischke MS, Sussman MR, Helariutta Y, Kakimoto T: Cytokinins regulate a bidirectional phosphorelay network in Arabidopsis. Curr Biol 2006, 16:1116-1122.

7. Imamura A, Hanaki N, Umeda H, Nakamura A, Suzuki T, Ueguchi C, Mizuno T: Response regulators implicated in His-to-Asp phosphotransfer signaling in Arabidopsis. Proc Natl Acad Sci U S A 1998, 95:2691-2696.

8. To JP, Deruère J, Maxwell BB, Morris VF, Hutchison CE, Ferreira FJ, Schaller GE, Kieber JJ: Cytokinin regulates type-a Arabidopsis response regulator activity and protein stability via two-component phosphorelay. Plant Cell 2007, 19:3901-3914.

9. Dortay H, Mehnert N, Bürkle L, Schmülling T, Heyl A: Analysis of protein interactions within the cytokinin-signaling pathway of Arabidopsis thaliana. FEBS J 2006, 273:4631-4644

10. Dortay H, Gruhn N, Pfeifer A, Schwerdtner M, Schmülling T, Heyl A: Toward an interaction map of the two-component signaling pathway of Arabidopsis thaliana. J Proteome Res 2008, 7:3649-3660.

11. Caesar K, Thamm AM, Witthöft J, Elgass K, Huppenberger P, Grefen C, Horak J, Harter K: Evidence for the localization of the Arabidopsis cytokinin receptors AHK3 and AHK4 in the endoplasmic reticulum. J Exp Bot 2011, 62:5571-5580.

12. Punwani JA, Hutchison CE, Schaller GE, Kieber JJ: The subcellular distribution of the Arabidopsis histidine phosphotransfer proteins is independent of cytokinin signaling. Plant J 2010, 62:473-482.

13. Veerabagu M, Elgass K, Kirchler T, Huppenberger P, Harter K, Chaban C, Mira-Rodado V: The Arabidopsis B-type response regulator 18 homomerizes and positively regulates cytokinin responses. Plant J 2012, 72:721-731.

14. Pils $B$, Heyl A: Unraveling the evolution of cytokinin signaling. Plant Physiol 2009, 151:782-791.

15. Ishida K, Yamashino T, Nakanishi H, Mizuno T: Classification of the genes involved in the two-component system of the moss Physcomitrella patens. Biosci Biotechnol Biochem 2010, 74:2542-2545.

16. Chapman BA, Bowers JE, Feltus FA, Paterson AH: Buffering crucial functions by paleologous duplicated genes may impart cyclicality to angiosperm genome duplication. Proc Natl Acad Sci USA 2006, 103:2730-2735

17. Wullschleger SD, Weston DJ, Difazio SP, Tuskan GA: Revisiting the sequencing of the first tree genome: populus trichocarpa. Tree Physiol 2013, 33:357-364

18. Tuskan GA, Difazio S, Jansson S, Bohlmann J, Grigoriev I, Hellsten U, Putnam N, Ralph S, Rombauts S, Salamov A, Schein J, Sterck L, Aerts A, Bhalerao RR, Bhalerao RP, Blaudez D, Boerjan W, Brun A, Brunner A, Busov V, Campbell M, Carlson J, Chalot M, Chapman J, Chen GL, Cooper D, Coutinho PM, Couturier J, Covert S, Cronk Q, et al: The genome of black cottonwood, populus trichocarpa (torr. \& Gray). Science 2006, 313:1596-1604.

19. Nieminen K, Immanen J, Laxell M, Kauppinen L, Tarkowski P, Dolezal K Tähtiharju S, Elo A, Decourteix M, Ljung K, Bhalerao R, Keinonen K, Albert VA, Helariutta Y: Cytokinin signaling regulates cambial development in poplar. Proc Natl Acad Sci USA 2008, 105:20032-20037.

20. Ramírez-Carvajal GA, Morse AM, Davis JM: Transcript profiles of the cytokinin response regulator gene family in populus imply diverse roles in plant development. New Phytol 2008, 177:77-89.

21. Sosinski B, Gannavarapu M, Beck LE, Rajapakse S, Ballard RE, Abbott AG Characterization of microsatellite markers in peach [Prunus persica (L.) Batsch]. Theor App/ Genet 2000, 101:421-428.

22. Arús P, Verde I, Sosinski B, Zhebentyayeva T, Abbott AG: The peach genome. Tree Genetics Genomes. 2012, 8:531-547.

23. Verde I, Abbott AG, Scalabrin S, Jung S, Shu S, Marroni F, Zhebentyayeva T, Dettori MT, Grimwood J, Cattonaro F, Zuccolo A, Rossini L, Jenkins J, Vendramin E, Meisel LA, Decroocq V, Sosinski B, Prochnik S, Mitros T, Policriti A, Cipriani G, Dondini L, Ficklin S, Goodstein DM, Xuan P, Fabbro CD, Aramini V, Copetti D, Gonzalez S, Horner DS, et al: The high-quality draft genome of peach (Prunus persica) identifies unique patterns of genetic diversity, domestication and genome evolution. Nat Genet 2013, 45:487-494

24. Arnau J, Tadeo F, Guerri J, Primo-Millo E: Cytokinins in peach: endogenous levels during early fruit development. Plant Physiol Biochem 1999, 37:741-750.

25. Zhang C, Whiting M: Improving "Bing" sweet cherry fruit quality with plant growth regulators. Sci Hort 2011, 127:341-346.

26. Ogata R, Saito T, Oshima K: Effect of N-phenyl-N-(4-pyridyl) urea on fruit size: apple, Japanese pear, grapevine and kiwifruit. Acta Hort. 1989, 239:395-398.

27. Tartarini S, Sansavini S, Ventura M: CPPU control of fruit morphogenesis in apple. Sci Hort 1993, 53:273-279.

28. Zhang C, Tanabe K, Wang S, Tamura F, Yoshida A, Matsumoto K: The impact of cell division and cell enlargement on the evolution of fruit size in Pyrus pyrifolia. Ann Bot 2006, 98:537-543.

29. Zhang $C$, Lee $U$, Tanabe $K$ : Hormonal regulation of fruit set, parthenogenesis induction and fruit expansion in Japanese pear. Plant Growth Regul 2008, 55:231-240.

30. Wang H, Moore MJ, Soltis PS, Bell CD, Brockington SF, Alexandre R, Davis CC, Latvis M, Manchester SR, Soltis DE: Rosid radiation and the rapid rise of angiosperm-dominated forests. Proc Natl Acad Sci USA 2009, 106:38533858.

31. Tang H, Bowers JE, Wang $X$, Ming R, Alam M, Paterson AH: Synteny and collinearity in plant genomes. Science 2008, 320:486-488.

32. Jung S, Cestaro A, Troggio M, Main D, Zheng P, Cho I, Folta KM, Sosinski B, Abbott A, Celton JM, Arús P, Shulaev V, Verde I, Morgante M, Rokhsar D, Velasco R, Sargent DJ: Whole genome comparisons of Fragaria, Prunus and Malus reveal different modes of evolution between Rosaceous subfamilies. BMC Genomics 2012, 4(13):129. doi: 10.1186/1471-2164-13-129.

33. Paterson $\mathrm{AH}$, Freeling $\mathrm{M}$, Tang $\mathrm{H}$, Wang $\mathrm{X}$ : Insights from the comparison of plant genome sequences. Annu Rev Plant Biol 2010, 61:349-372. doi: 10.1146/annurev-arplant-042809-112235. 
34. Jung S, Staton M, Lee T, Blenda A, Svancara R, Abbott A, Main D: GDR (Genome Database for Rosaceae): integrated web-database for Rosaceae genomics and genetics data. Nucleic Acids Res 2008, 36(Database issue):D1034-D1040.

35. Grigoriev IV, Nordberg H, Shabalov I, Aerts A, Cantor N, Goodstein D, Kuo A, Minovitsky S, Nikitin R, Ohm RA, Otillar R, Poliakov A, Ratnere I, Riley R, Smirnova T, Rokhsar D, Dubchak I: The genome portal of the department of energy joint genome institute I. V. Nucleic Acids Res 2012, 40(database issue):D26-D32.

36. Goodstein D, Shu S, Howson R, Neupane R, Hayes R, Fazo J, Mitros T, Dirks W, Hellsten W, Putnam N, Rokhsar D: Phytozome: a comparative platform for green plant genomics. Nucleic Acids Res 2012, 40(D1):D1178-D1186.

37. Lamesch P, Berardini T, Li D, Swarbreck D, Wilks C, Sasidharan R, Muller R Dreher K, Alexander D, Garcia-Hernandez M, Karthikeyan A, Lee C, Nelson W, Ploetz L, Singh S, Wensel A, Huala: The Arabidopsis information resource (TAIR): improved gene annotation and new tools. Nucleic Acids Res 2011:1-9. doi: 10.1093/nar/gkr1090.

38. Hulsen T, Huynen M, de Vlieg J, Groenen M: Benchmarking ortholog identification methods using functional genomics data. Genome Biol 2006, 7:R31.

39. Waterhouse AM, Procter JB, Martin DM, Clamp M, Barton GJ: Jalview version 2 a multiple sequence alignment editor and analysis workbench. Bioinformatics 2009, 25:1189-1191. doi: 10.1093/bioinformatics/btp033.

40. Edgar RC: MUSCLE: multiple sequence alignment with high accuracy and high throughput. Nucleic Acids Res 2004, 32:1792-1797.

41. Stamatakis $A$, Hoover $P$, Rougemont J: A rapid bootstrap algorithm for the RAxML web servers. Syst Biol 2008, 57:758-771.

42. Werner T, Motyka V, Strnad M, Schmülling T: Regulation of plant growth by cytokinin. Proc Natl Acad Sci U S A 2001, 98:10487-10492.

43. Werner T, Motyka V, Laucou V, Smets R, Van Onckelen H, Schmülling T: Cytokinin deficient transgenic Arabidopsis plants show multiple developmental alterations indicating opposite functions of cytokinins in the regulation of shoot and root meristem activity. Plant Cell 2003, 15:2532-2550

44. Schmülling T, Werner T, Riefler M, Krupková E, Bartrina y Manns I: Structure and function of cytokinin oxidase/dehydrogenase genes of maize, rice, Arabidopsis and other species. J Plant Res 2003, 116:241-252.

45. Kakimoto T: Identification of plant cytokinin biosynthesis enzymes as dimethylallyl diphosphate: ATP/ADP isopentenyltransferases. Plant Cell Physiol 2001, 42:677-685.

46. Takei K, Sakakibara H, Taniguchi M, Sugiyama T: Nitrogen-dependent accumulation of cytokinins in root and the translocation to leaf: Implication of cytokinin species that induces gene expression of maize response regulator. Plant Cell Physiol 2001, 42:85-93.

47. Miyawaki K, Matsumoto-Kitano M, Kakimoto T: Expression of cytokinin biosynthetic isopentenyltransferase genes in Arabidopsis: tissue specificity and regulation by auxin, cytokinin, and nitrate. Plant J 2004, 37:128-138

48. Miyawaki K, Tarkowski P, Matsumoto-Kitano M, Kato T, Sato S, Tarkowska D, Tabata S, Sandberg G, Kakimoto T: Roles of Arabidopsis ATP/ADP isopentenyltransferases and tRNA isopentenyltransferases in cytokinin biosynthesis. Proc Natl Acad Sci U S A 2006, 103:16598-16603.

49. Matsumoto-Kitano M, Kusumoto T, Tarkowski P, Kinoshita-Tsujimura K, Václavíková K, Miyawaki K, Kakimoto T: Cytokinins are central regulators of cambial activity. Proc Natl Acad Sci U S A 2008, 105:20027-20031.

50. Kuroha T, Tokunaga H, Kojima M, Ueda N, Ishida T, Nagawa S, Fukuda H, Sugimoto K, Sakakibara H: Functional analyses of LONELY GUY cytokinin-activating enzymes reveal the importance of the direct activation pathway in Arabidopsis. Plant Cell 2009, 21:3152-3169.

51. Tokunaga H, Kojima M, Kuroha T, Ishida T, Sugimoto K, Kiba T, Sakakibara H: Arabidopsis lonely guy (LOG) multiple mutants reveal a central role of the LOG-dependent pathway in cytokinin activation. Plant J 2012, 69:355-365

52. Kurakawa T, Ueda N, Maekawa M, Kobayashi K, Kojima M, Nagato Y, Sakakibara H, Kyozuka J: Direct control of shoot meristem activity by a cytokinin-activating enzyme. Nature 2007, 445:652-655.

53. Chickarmane VS, Gordon SP, Tarr PT, Heisler MG, Meyerowitz EM: Cytokinin signaling as a positional cue for patterning the apical-basal axis of the growing Arabidopsis shoot meristem. Proc Natl Acad Sci USA 2012, 109:4002-4007.
54. Higuchi M, Pischke MS, Mähönen AP, Miyawaki K, Hashimoto Y, Seki M, Kobayashi M, Shinozaki K, Kato T, Tabata S, Helariutta Y, Sussman MR, Kakimoto T: In planta functions of the Arabidopsis cytokinin receptor family. Proc Natl Acad Sci USA 2004, 101:8821-8826.

55. Kakimoto T: CKI1, a histidine kinase homolog implicated in cytokinin signal transduction. Science 1996, 274:982-985.

56. Hwang I, Chen HC, Sheen J: Two-component signal transduction pathways in Arabidopsis. Plant Physiol 2002, 129:500-515.

57. Kakimoto T: Perception and signal transduction of cytokinins. Annu Rev Plant Biol 2003, 54:605-627.

58. Iwama A, Yamashino T, Tanaka Y, Sakakibara H, Kakimoto T, Sato S, Kato T, Tabata S, Nagatani A, Mizuno T: AHK5 histidine kinase regulates root elongation through an ETR1-dependent abscisic acid and ethylene signaling pathway in Arabidopsis thaliana. Plant Cell Physiol 2007, 48:375-380.

59. Mähönen AP, Bonke M, Kauppinen L, Riikonen M, Benfey N, Helariutta Y: A novel two-component hybrid molecule regulates vascular morphogenesis of the Arabidopsis root. Genes Dev 2000, 14:2938-2943.

60. Yamada H, Suzuki T, Terada K, Takei K, Ishikawa K, Miwa K, Yamashino T, Mizuno T: The Arabidopsis AHK4 histidine kinase is a cytokinin-binding receptor that transduces cytokinin signals across the membrane. Plant Cell Physiol 2001, 42:1017-1023.

61. Deng $Y$, Dong $H$, Mu J, Ren B, Zheng B, Ji Z, Yang WC, Liang Y, Zuo J: Arabidopsis histidine kinase CKI1 acts upstream of histidine phosphotransfer proteins to regulate female gametophyte development and vegetative growth. Plant Cell 2010, 22:1232-1248.

62. Pekárová B, Klumpler T, Třísková O, Horák J, Jansen $\mathrm{S}$, Dopitová R, Borkovcová P, Papoušková V, Nejedlá E, Sklenář V, Marek J, Zídek L, Hejátko $J$, Janda L: Structure and binding specificity of the receiver domain of sensor histidine kinase CKI1 from Arabidopsis thaliana. Plant J 2011, 67:827-839

63. Wulfetange K, Lomin SN, Romanov GA, Stolz A, Heyl A, Schmülling T: The cytokinin receptors of Arabidopsis are located mainly to the endoplasmic reticulum. Plant Physiol 2011, 156:1808-1818.

64. Hejátko J, Ryu H, Kim GT, Dobesová R, Choi S, Choi SM, Soucek P, Horák J, Pekárová B, Palme K, Brzobohaty B, Hwang I: The histidine kinases CYTOKININ-INDEPENDENT1 and ARABIDOPSIS HISTIDINE KINASE2 and 3 regulate vascular tissue development in Arabidopsis shoots. Plant Cell 2009, 21:2008-2021.

65. Mochida K, Yoshida T, Sakurai T, Yamaguchi-Shinozaki K, Shinozaki K, Tran LS: Genome-wide analysis of two-component systems and prediction of stress-responsive two-component system members in soybean. DNA Res 2010, 17:303-324.

66. Ito $\mathrm{Y}$, Kurata $\mathrm{N}$ : Identification and characterization of cytokinin-signalling gene families in rice. Gene 2006, 382:57-65

67. Pischke MS, Jones LG, Otsuga D, Fernandez DE, Drews GN, Sussman MR: An Arabidopsis histidine kinase is essential for megagametogenesis. Proc Natl Acad Sci USA 2002, 99:15800-15805.

68. Suzuki T, Ishikawa K, Yamashino T, Mizuno T: An Arabidopsis histidinecontaining phosphotransfer (HPt) factor implicated in phosphorelay signal transduction: overexpression of AHP2 in plants results in hypersensitiveness to cytokinin. Plant Cell Physiol 2002, 43:123-129.

69. Miyata S, Urao T, Yamaguchi-Shinozaki K, Shinozaki K: Characterization of genes for two-component phosphorelay mediators with a single HPt domain in Arabidopsis thaliana. FEBS Lett 1998, 437:11-14.

70. Suzuki T, Imamura A, Ueguchi C, Mizuno T: Histidine-containing phosphotransfer (HPt) signal transducers implicated in His-to-Asp phosphorelay in Arabidopsis. Plant Cell Physiol 1998, 39:1258-1268.

71. Suzuki T, Sakurai K, Imamura A, Nakamura A, Ueguchi C, Mizuno T: Compilation and characterization of histidine-containing phosphotransmitters implicated in His-to-Asp phosphorelay in plants: AHP signal transducers of Arabidopsis thaliana. Biosci Biotechnol Biochem 2000, 64:2486-2489.

72. Tanaka Y, Suzuki T, Yamashino T, Mizuno T: Comparative studies of the AHP histidine-containing phosphotransmitters implicated in His-to-Asp phosphorelay in Arabidopsis thaliana. Biosci Biotechol. Biochem 2004, 68:462-465.

73. Hutchison CE, Li J, Argueso C, Gonzalez M, Lee E, Lewis MW, Maxwell BB, Perdue TD, Schaller GE, Alonso JM, Ecker JR, Kieber JJ: The Arabidopsis histidine phosphotransfer proteins are redundant positive regulators of cytokinin signaling. Plant Cell 2006, 18:3073-3087. 
74. Mähönen AP, Bishopp A, Higuchi M, Nieminen KM, Kinoshita K, Törmäkangas K, Ikeda Y, Oka A, Kakimoto T, Helariutta Y: Cytokinin signaling and its inhibitor AHP6 regulate cell fate during vascular development. Science 2006, 311:94-98.

75. Tsai YC, Weir NR, Hill K, Zhang W, Kim HJ, Shiu SH, Schaller GE, Kieber JJ: Characterization of genes involved in cytokinin signaling and metabolism from rice. Plant Physiol 2012, 158:1666-1684.

76. Chu ZX, Ma Q, Lin YX, Tang XL, Zhou YQ, Zhu SW, Fan J, Cheng BJ: Genome-wide identification, classification, and analysis of twocomponent signal system genes in maize. Genet Mol Res 2011, 10:3316-3330

77. Jung KW, Oh SI, Kim YY, Yoo KS, Cui MH, Shin JS: Arabidopsis histidine-containing phosphotransfer factor 4 (AHP4) negatively regulates secondary wall thickening of the anther endothecium during flowering. Mol Cells 2008, 25:294-300.

78. Kiba T, Aoki K, Sakakibara H, Mizuno T: Arabidopsis response regulator, ARR22, ectopic expression of which results in phenotypes similar to the wol cytokinin-receptor mutant. Plant Cell Physiol 2004, 45:1063-1077.

79. Makino S, Kiba T, Imamura A, Hanaki N, Nakamura A, Suzuki T, Taniguchi M, Ueguchi C, Sugiyama T, Mizuno T: Genes encoding pseudo-response regulators: insight into His-Asp phosphorelay and circadian rhythm in Arabidopsis thaliana. Plant Cell Physiol 2000, 41:791-803.

80. Mizuno T: Plant response regulators implicated in signal transduction and circadian rhythm. Curr Opin Plant Biol 2004, 7:499-505.

81. Murakami M, Yamashino T, Mizuno T: Characterization of circadianassociated APRR3 pseudo-response regulator belonging to the APRR1/ TOC1 quintet in Arabidopsis thaliana. Plant Cell Physiol 2004, 45:645-650.

82. Sakai H, Honma T, Aoyama T, Sato S, Kato T, Tabata S, Oka A: ARR1, a transcription factor for genes immediately responsive to cytokinins. Science 2001, 294:1519-1521.

83. Imamura A, Kiba T, Tajima Y, Yamashino T, Mizuno T: In vivo and in vitro characterization of the ARR11 response regulator implicated in the His-to-Asp phosphorelay signal transduction in Arabidopsis thaliana. Plant Cell Physiol 2003, 44:122-131.

84. Mason MG, Mathews DE, Argyros DA, Maxwell BB, Kieber JJ, Alonso JM, Ecker JR, Schaller GE: Multiple type-B response regulators mediate cytokinin signal transduction in Arabidopsis. Plant Cell 2005, 17:3007-3018.

85. Kim K, Ryu H, Cho YH, Scacchi E, Sabatini S, Hwang I: Cytokinin-facilitated proteolysis of ARABIDOPSIS RESPONSE REGULATOR 2 attenuates signaling output in two-component circuitry. Plant J 2012, 69:934-945

86. Ishida K, Yamashino T, Yokoyama A, Mizuno T: Three type-B response regulators, ARR1, ARR10 and ARR12, play essential but redundant roles in cytokinin signal transduction throughout the life cycle of Arabidopsis thaliana. Plant Cell Physiol 2008, 49:47-57.

87. Argyros RD, Mathews DE, Chiang YH, Palmer CM, Thibault DM, Etheridge $N$ Argyros DA, Mason MG, Kieber JJ, Schaller GE: Type B response regulators of Arabidopsis play key roles in cytokinin signaling and plant development. Plant Cell 2008, 20:2102-2116.

88. Tajima Y, Imamura A, Kiba T, Amano Y, Yamashino T, Mizuno T: Comparative studies on the type-B response regulators revealing their distinctive properties in the His-to-Asp phosphorelay signal transduction of Arabidopsis thaliana. Plant Cell Physiol 2004, 45:28-39.

89. Lohrmann J, Buchholz G, Keitel C, Sweere U, Kircher S, Bäurle I, Kudla J, Schäfer E, Harter K: Differential expression and nuclear localization of response regulator-like proteins from Arabidopsis thaliana. Plant Biol 1999, 1:495-505.

90. Rashotte AM, Carson SD, To JP, Kieber JJ: Expression profiling of cytokinin action in Arabidopsis. Plant Physiol 2003, 132:1998-2011.

91. To JPC, Haberer G, Ferreira FJ, Deruère J, Mason MG, Schaller GE, Alonso JM, Ecker JR, Kieber JJ: Type-A Arabidopsis response regulators are partially reduntant negative regulators of cytokinin signaling. Plant Cell 2004, 16:658-671.

92. Buechel S, Leibfried A, To JP, Zhao Z, Andersen SU, Kieber JJ, Lohmann JU: Role of A-type ARABIDOPSIS RESPONSE REGULATORS in meristem maintenance and regeneration. Eur J Cell Biol 2010, 89:279-284.

93. Zhang W, To JP, Cheng CY, Eric Schaller G, Kieber JJ: Type-A response regulators are required for proper root apical meristem function through post-transcriptional regulation of PIN auxin efflux carriers. Plant J 2011, 68:1-10.

94. Kim J: Phosphorylation of A-Type ARR to function as negative regulator of cytokinin signal transduction. Plant Signal Behav 2008, 3:348-350.
95. Horák J, Grefen C, Berendzen KW, Hahn A, Stierhof YD, Stadelhofer B, Stahl $M$, Koncz C, Harter K: The Arabidopsis thaliana response regulator ARR22 is a putative AHP phospho-histidine phosphatase expressed in the chalaza of developing seeds. BMC Plant Biol 2008, 8:77.

96. Gattolin S, Alandete-Saez M, Elliott K, Gonzalez-Carranza Z, Naomab E, Powell C, Roberts JA: Spatial and temporal expression of the response regulators ARR22 and ARR24 in Arabidopsis thaliana. J Exp Bot 2006, 57:4225-4233.

doi:10.1186/1471-2164-14-885

Cite this article as: Immanen et al:: Characterization of cytokinin signaling and homeostasis gene families in two hardwood tree species: Populus trichocarpa and Prunus persica. BMC Genomics 2013 14:885.

\section{Submit your next manuscript to BioMed Central and take full advantage of:}

- Convenient online submission

- Thorough peer review

- No space constraints or color figure charges

- Immediate publication on acceptance

- Inclusion in PubMed, CAS, Scopus and Google Scholar

- Research which is freely available for redistribution

Submit your manuscript at www.biomedcentral.com/submit
C) Biomed Central 